\title{
Mushroom-bodies mediate hierarchical interactions between fact- and skill-learning in Drosophila
}

\author{
Running head: Mushroom-bodies regulate habit formation
}

\author{
Björn Brembs ${ }^{1}$ and Wolfgang Plendl ${ }^{2}$
}

\begin{abstract}
1 For correspondence: Freie Universität Berlin, Institut für Biologie - Neurobiologie, Königin-Luise Str. 28/30, 14195 Berlin, Germany. Email: bjoern@brembs.net, phone: +49 (0)30 838 55050, fax: +49 (0)30 83855455

2 Lehrstuhl für Neurobiologie und Genetik, Biozentrum, Universität Würzburg, Germany.
\end{abstract}

\section{Summary}

Different brain circuits mediate the acquisition of skills and habits (via operant/instrumental learning) and the acquisition of facts (via classical/Pavlovian learning). Realistic learning situations always comprise interactions of skill- and fact-learning components (composite learning). So far, these interactions have escaped thorough scrutiny. Fixed flying Drosophila melanogaster at the torque meter provide one of the very few systems where the relationship of operant and classical predictors in composite learning can be studied with sufficient rigor. Experiments with wildtype, mutant and transgenic flies show that there is an interaction between predictive stimuli (classical component) and goal-directed actions (operant component) which makes composite conditioning more effective than the operant and classical components alone. Rutabaga (rut) mutants are impaired in learning about the (classical) stimuli, but show improved (operant) behavior learning. This is the first evidence that operant and classical conditioning differ not only at the circuit, but also at the molecular level. The interaction between operant and classical components is reciprocal and hierarchical, such that an impaired classical component (in rut flies) suppresses retrieval and an intact classical component suppresses acquisition of the operant component. Experiments with transgenic flies demonstrate that this suppression of operant acquisition is mediated by the mushroom-bodies and serves to ensure that the classical memories can be generalized for access by other behaviors. Extended training can overcome this suppression and transforms goal-directed actions into habitual responses. In conclusion, composite conditioning consists of two components with reciprocal, hierarchical interactions. Acquisition of the rut-dependent classical component suppresses acquisition of the rut-independent operant component via the mushroom-bodies. The operant component facilitates acquisition of the classical component via unknown, non-mushroom-body pathways. This interaction leads to efficient learning, enables generalization and prevents premature habit-formation. Habit formation after extended training reveals the gate-keeping role of the mushroom-bodies, allowing only wellrehearsed behaviors to consolidate into habits. 